\title{
A HAEMORRHAGIC DISORDER IN PREGNANCY DUE TO AN "ANTICOAGULANT" PREVENTING THE CONVERSION OF FIBRINOGEN TO FIBRIN
}

\author{
BY \\ S. J. BAKER AND E. JACOB \\ From the Department of Haematology, Christian Medical College Hospital, Vellore
}

(RECEIVED FOR PUBLICATION SEPTEMBER 3, 1959)

\begin{abstract}
A bleeding disorder occurring during labour and affecting mother and foetus is described.
All stages of coagulation were normal until the reaction fibrinogen $\rightarrow$ fibrin. Either there was some abnormality present in the fibrinogen molecule, or there was an " anticoagulant" acting at this stage.

The abnormality was reversible by protamine sulphate and toluidine blue, but in other respects did not resemble hyperheparinaemia.

Reversibility of a clotting defect by protamine or toluidine blue is not sufficient evidence on which to make a diagnosis of hyperheparinaemia.

Demonstration of a prolonged clotting time occurring in late pregnancy does not necessarily justify the diagnosis of " afibrinogenaemia."
\end{abstract}

Severe haemorrhagic disorders occurring towards the end of pregnancy have usually been found to be associated with hypo- or afibrinogenaemia (Dieckmann, 1936; Moore, 1954). A few cases of haemorrhagic disorder associated with the presence of an anticoagulant have also been described following pregnancy; for example, the cases described by Dreskin and Rosenthal (1950) and by Biggs and Macfarlane (1957), in which the anticoagulant concerned inactivated antihaemophilic globulin.

This report concerns a fatal haemorrhagic diathesis which developed in an otherwise healthy woman in the 38th week of pregnancy. The disorder was at first thought to be due to afibrinogenaemia, but on further investigation it was shown to be due to the presence of an "anticoagulant" which prevented the conversion of fibrinogen to fibrin.

\section{Methods}

The following investigations were carried out by the methods described by Biggs and Macfarlane (1957): Bleeding time (Ivy), Hess test, whole blood clotting time (Lee and White), recalcification clotting time, one-stage prothrombin time using human brain thromboplastin (Quick), two-stage prothrombin time, and thromboplastin generation test.

Haemoglobin estimation, white cell and platelet count (direct), and thrombin generation tests were carried out by the methods described by Dacie (1956). Fibrinogen estimations were performed by the microKjeldahl method.

Human "heparin" was prepared from liver obtained at necropsy performed half an hour after death. The method followed was the same as given in the British Pharmacopoeia (General Medical Council, 1948) except that cetavlon was employed instead of brucine in the final purification. This resulted in a preparation which had definite anticoagulant properties which were reversible by protamine sulphate and toluidine blue. A further study of the properties and composition of this substance is being undertaken.

\section{Case Report}

A 35-year-old married woman, at term with her fifth pregnancy, was admitted to the obstetric department of the Christian Medical College Hospital. Her four previous pregnancies had all been uneventful. There was no history of any previous bleeding episode, and her menstrual periods had always been normal. The patient had had no serious illness in the past, and had not had any drugs or medicine recently.

Physical examination revealed an apparently healthy woman. The blood pressure was $100 \mathrm{~mm}$. of mercury systolic and $60 \mathrm{~mm}$. diastolic. The fundus was at 38 weeks' height, and the foetal head was floating with the vertex presenting. Uterine contractions were occurring once every five to eight minutes. The foetal heart sounds were normal and the rate was 144 per minute. 
The patient's haemoglobin was 7.2 g. per $100 \mathrm{ml}$. and the blood film showed a moderate degree of hypochromia of the red cells. Platelets were present in the blood film in normal numbers. The urine contained a trace of albumin, but was otherwise normal.

The patient remained well for the next 48 hours after admission and had no further uterine contractions. On the evening of the second day after admission she developed fever with a temperature of $102^{\circ}$, and complained of mild headache. Clinical examination at this time revealed no cause for this fever, except that a centrifuged deposit of the urine contained $20-30$ pus cells per high-power field. A blood examination showed a white cell count of 16,000 per c.mm. (82\% polymorphs, $3 \%$ eosinophils, and $15 \%$ lymphocytes). The patient was given 10 grains of aspirin, and intramuscular penicillin therapy $(600,000$ units of procaine penicillin 12-hourly) was begun. On the morning of the third day at about 7.30 labour started again. At 10 a.m. the foetal heart sounds were noted to be irregular, and liquor amnii heavily stained with meconium was passed. By 3 p.m. the cervix was three-fifths dilated, but the foetal heart could no longer be heard. At this stage it was noted that the patient had a little bleeding from the gums, but the full significance of this was not realized. At 4 p.m. the bleeding from the gums had increased, and it was also noted that the patient was bleeding from a venepuncture wound, and that there was a moderate amount of bleeding per vaginam. Blood was taken for various coagulation investigations, and a blood transfusion with freshly drawn bank blood was started. At this stage it was suspected that this was probably a case of afibrinogenaemia, and this was apparently confirmed by the demonstration of a prolonged whole blood clotting time. As no fibrinogen or concentrated plasma was available, the only possible treatment was the transfusion of fresh blood. However, in spite of transfusion bleeding continued.

By 5 p.m. it had been shown in vitro that the blood, which was apparently incoagulable, could be made to clot by the addition of protamine sulphate. This suggested a diagnosis of hyperheparinaemia, and therefore an injection of $150 \mathrm{mg}$. of protamine sulphate was given intravenously as a $1 \%$ solution at 5.12 p.m. Unfortunately this was all the protamine sulphate available in the hospital and no more could be obtained immediately. Three minutes after the injection a further specimen of blood was taken for coagulation studies. Following the injection bleeding appeared to stop for about 20 minutes, but after this bleeding from the vagina, the gums, and various needle puncture wounds recurred. Blood transfusion was continued while further efforts were made to obtain protamine sulphate or toluidine blue, but unfortunately neither was readily available.

At 7 p.m. a stillborn foetus was delivered, and blood was taken from the umbilical vein for examination. At 7.15 p.m. the placenta was expelled and the bleeding from the vagina increased in severity. An intravenous injection of ergometrine was given, but it made little difference to the severity of bleeding. Blood transfusion was continued throughout, at times under pressure, in an endeavour to keep up with the blood losses. At 7.45 p.m. a solution of toluidine blue, $1 \mathrm{~g}$. in $500 \mathrm{ml}$. of $5 \%$ glucose, became available, and was started as a rapid intravenous drip via a second vein. However, in spite of all therapy the patient's condition rapidly deteriorated, she developed peripheral circulatory failure and pulmonary oedema and died at 8.15 p.m. At the time of death it was noticed that the most recent blood from the vagina was clotting spontaneously. This was the first time that this had been noted. Immediately after death, blood was taken from the heart for further tests.

\section{Post-mortem Examination}

Post-mortem examination of the mother showed bluish staining of all the tissues in the body. This was most marked on the internal surface of the aorta, the inside of the renal pelves and ureters, and in the brain.

The trachea and bronchi contained a lot of bloodstained frothy fluid. There were some petechial haemorrhages in the wall of the aorta, otherwise the cardiovascular system was normal. There was some altered blood in the stomach, with patchy areas of petechial haemorrhage all over the mucosa. Both liver and spleen were congested, but otherwise normal. The kidneys were normal, except that there were numerous subcapsular petechial haemorrhages. The bladder also showed some small haemorrhages. The upper two-thirds of the body of the uterus was firm and well contracted, but the lower portion was soft and flabby with marked haemorrhage into the muscle. The placenta had been attached anteriorly at the junction of the upper and lower uterine segments. No abnormality was present in the central nervous system.

Post-mortem examination of the foetus showed no abnormality, except for atelectasis of the lungs, some congestion of the liver, and haemorrhage into the cortex of both adrenal glands.

Tissue sections from the various organs and from the skin, and bone marrow smears from both mother and foetus stained with toluidine blue failed to reveal any evidence of increase in the number of mast cells.

\section{Investigation of Coagulation Defect in Maternal Blood}

Except where otherwise specified investigations were performed on blood taken at 4 p.m. before any treatment had been given.

Bleeding Time-Bleeding time at 4 p.m. was 15 minutes.

Hess Test.-The Hess test was negative at 4.10 p.m.

Platelet Count.-The platelet count was 60,000 per c.mm. This was confirmed by the examination of a stained blood film which showed a decrease in the number of platelets. 
Bone Marrow.-The marrow fragments were slightly hyperplastic. The developing red and white cells and the megakaryocytes were normal.

Clotting Time-The clotting time of whole blood was as follows:

Initial sample taken at 4 p.m. No clot formed after 24 hours. In a sample taken at 5.15 p.m., three minutes after $150 \mathrm{mg}$. of protamine sulphate had been given, a few threads of fibrin appeared after one hour.

In a sample taken immediately after death the clotting time was 10 minutes. This formed a good solid clot which gelled the whole of the blood, and retracted normally.

Effect of Addition of Protamine Sulphate in Clotting Time of Plasma.-The addition of protamine sulphate, $10 \mathrm{mg}$., to $1 \mathrm{ml}$. of uncitrated plasma resulted in the rapid formation of a network of fibrin. As the amount of protamine sulphate added was decreased, the time at which the fibrin appeared was prolonged, the amount of fibrin formed decreased, and the nature of the fibrin changed from a network of fibrin strands to a few fine granules of fibrin. When the amount of protamine added was sufficiently decreased no fibrin was formed at all (Fig. 1). Similar results were found with the addition of toluidine blue.

\begin{tabular}{|c|c|c|c|c|c|c|c|c|}
\hline $\begin{array}{l}\text { patient's } \\
\text { plasma }\end{array}$ & 1 & 1 & 1 & 1 & 1 & I & I & 1 \\
\hline $\begin{array}{c}\text { protamine } \\
\text { mgm. }\end{array}$ & 0 & 10 & 5 & 2.5 & $1 \cdot 2$ & 0.6 & 0.3 & 0.15 \\
\hline $\begin{array}{c}\text { clotting time } \\
\text { seconds }\end{array}$ & $\infty$ & 1 & 5 & 18 & 25 & 40 & $\infty$ & $\infty$ \\
\hline size of clot & & $\bigsqcup^{1}$ & 11 & & & & & \\
\hline
\end{tabular}

FIG. 1.-The clotting time and the size and nature of the clot produced, when varying amounts of protamine sulphate were added to the patient's uncitrated plasma. In each case the protamine was made up to $0.5 \mathrm{ml}$. with normal saline and added to $1 \mathrm{ml}$. of plasma.

Recalcification Time of Normal Plasma on Addition of Patient's Plasma.-The addition of the patient's plasma to normal plasma in equal volumes caused only a slight increase in the recalcification clotting time. As the amount of patient's plasma relative to normal plasma was increased the recalcification time became more prolonged (Fig. 2), and the size of the clot formed progressively decreased, suggesting that the fibrinogen from the patient's plasma did not contribute significantly to the fibrin formed.

Quick's One-stage Prothrombin Time.-The one-stage prothrombin time showed no clot after four hours.

\begin{tabular}{|c|c|c|c|c|c|c|c|c|c|c|c|}
\hline $\begin{array}{l}\text { control } \\
\text { plosmo }\end{array}$ & 0 & 1 & 2 & 3 & 4 & 5 & 6 & 7 & 8 & 9 & 10 \\
\hline $\begin{array}{c}\text { potient's } \\
\text { plasmo }\end{array}$ & 10 & 9 & 8 & 7 & 6 & 5 & 4 & 3 & 2 & 1 & 0 \\
\hline $\begin{array}{l}\text { clotting time } \\
\text { seconds }\end{array}$ & $\infty$ & $\infty$ & 615 & 205 & 190 & 175 & 170 & 165 & 155 & 150 & 150 \\
\hline size of clot & & & & & & & & $\bigsqcup_{(2)}$ & 感 & W & 哃 \\
\hline
\end{tabular}

FIG. 2.-The recalcification clotting time and the size and nature of the clot produced with varying mixtures of control and patient's plasma.

Two-stage Prothrombin Time.-As measured by the area under the graph, this gave a result equivalent to $92 \%$ of a normal control.

Plasma "Fibrinogen."-When the plasma was clotted by the addition of protamine sulphate, the amount of fibrin formed from fibrinogen was 210 $\mathrm{mg}$. per $100 \mathrm{ml}$. of plasma.

Thrombin Generation Test. - Thrombin generation tests were carried out using the patient's platelet-rich plasma and a normal control plasma. The platelet count of the control plasma was adjusted by mixing "high spun" platelet-poor plasma with " low spun" platelet-rich plasma until the platelet count was approximately equal to that of the patient's plasma, viz., 120,000 per c.mm. The results of the thrombin generation test are shown in Fig. 3. It will be seen that the patient's plasma generated thrombin in a manner exactly comparable to normal control plasma with the same platelet count.

The results of the thrombin generation test on the patient's plasma were then compared with thrombin generation tests performed on the control

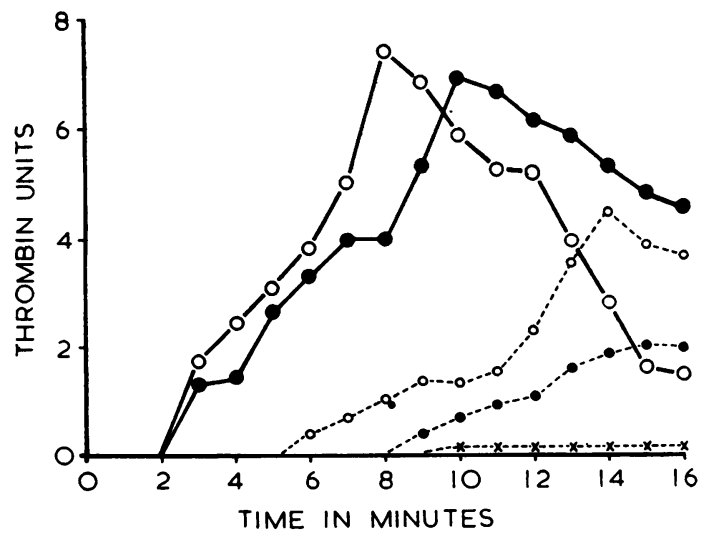

Fig. 3.-Results of thrombin generation tests: $0-0$ Normal control. Patient. O- - O Control + heparinclotting time 20 minutes. - - Control theparin-clotting time 40 minutes. $\times---X$ Control +heparin-infinite clotting time. 
plasma to which varying amounts of heparin* had been added. When sufficient heparin was added to the control plasma to make the clotting time greater than 12 hours no thrombin production could be demonstrated in the thrombin generation test. Decreasing the amount of heparin added caused increasing amounts of thrombin to appear, but the peak of the thrombin production was depressed and delayed (Fig. 3).

Similarly, when sufficient human "heparin" was added to the control plasma to render it incoagulable, there was no detectable thrombin formation in the thrombin generation test.

Thromboplastin Generation.-The thromboplastin generation test was carried out using the patient's barium sulphate adsorbed plasma, "serum" obtained after the patient's uncitrated blood had been kept for 12 hours at $37^{\circ} \mathrm{C}$., and a platelet suspension from a normal control. The

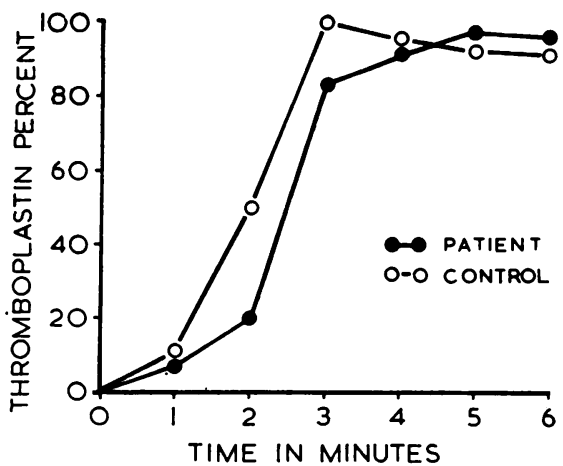

FIG. 4.-Results of thromboplastin generation tests. 0 - O Control. Patient.

results were identical with the thromboplastin generation when control-adsorbed plasma, control serum, and the same suspension of platelets were used (Fig. 4).

Fibrinolysin.-A clot from normal blood suspended in the patient's plasma did not undergo any detectable lysis when the two were incubated for 12 hours.

Attempted Transfer of the "Anticoagulant."Protamine sulphate was added to the patient's plasma drop by drop until fibrin was no longer precipitated. The fibrin was then removed by centrifugation and nucleic acid added to the supernatant to combine with the protamine, and the precipitate so formed was then removed by centrifugation. The resultant "serum" was then added to normal citrated plasma and the recalcification time measured. The addition of this "serum" did not cause any significant prolongation of the clotting time of the normal plasma.

\section{Investigation of Foetal Blood}

Clotting Time.-No clot was formed after 24 hours. As with the maternal blood samples, the clotting defect was corrected by the addition of protamine sulphate and toluidine blue.

One-stage Prothrombin Time.-No clot was formed after four hours.

Further investigations on the foetal blood were not possible owing to the small amount of blood available.

\section{Discussion}

Circulating Anticoagulants in Pregnancy.Several authors have reported haemorrhagic disorders associated with an anticoagulant occurring weeks or months after pregnancy, e.g., Madison and Quick (1945), Dreskin and Rosenthal (1950), Biggs and Macfarlane (1957). However, these cases had normal one-stage prothrombin times and had anticoagulants which appeared to act in the earlier stages of coagulation. The cases described in the latter two papers had an anticoagulant which was shown to be active against antihaemophilic globulin, and therefore differ from the present case.

Heparin-like Anticoagulants. - Jaques and Waters (1941) showed that a considerable rise in blood heparin occurred in dogs suffering from anaphylactic shock. Allen, Sanderson, Milham, Kirschon, and Jacobson (1948) found that the lengthening of the coagulation time which was seen in dogs exposed to ionizing radiation could be corrected by the addition of toluidiñe blue and protamine, and postulated that the cause of the haemorrhagic disorder was hyperheparinaemia. Castex (1946) and Castex and Pavlovsky (1947) reported a patient who had an anticoagulant which in some respects resembled heparin. Conley, Hartmann, and Morse (1949) described one patient in whom there was a heparin-like anticoagulant present in the plasma. Crisalli and Cotellessa (1951) described a 10-year-old boy with a haemorrhagic diathesis in which the clotting defect was corrected by toluidine blue and protamine. Bell (1951) reported a patient who had an anticoagulant which had both antithromboplastic and antithrombic activity, and was neutralized by the addition of protamine.

Physiological Action of Heparin.-Heparin interferes with the normal clotting mechanism in several ways. Howell and Holt (1918) found that the presence of heparin in the blood prevented the 
conversion of prothrombin to thrombin, and consluded that heparin was an antiprothrombin. Mellanby (1934) found that heparin inhibited the thrombin-fibrinogen reaction, and Quick (1938) showed that a component from the plasma albumin was necessary for this inhibitory effect. Klein and Seegers (1950) showed that heparin promoted the adsorption of thrombin by fibrin. Lyttleton (1950) showed that heparin, in association with a fraction of the plasma albumin, combined with thrombin, thus reducing its ability to clot fibrinogen. He also showed that the combination of heparin and thrombin is reversed by toluidine blue. Biggs, Douglas, and Macfarlane (1953) showed that heparin inhibited the formation of blood thromboplastin and suggested that this is the reason for the failure of prothrombin conversion in heparinized blood.

Resemblance of Clotting Defect to Hyperheparinaemia.-It is interesting that although the clotting defect in our patient was reversed by toluidine blue and protamine, and so resembled hyperheparinaemia, in all other respects it differed in its action from the recognized properties of heparin. As shown by the thromboplastin generation test the formation of blood thromboplastin was not inhibited. Similarly the thrombin generation test showed that the "anticoagulant" did not interfere with the conversion of prothrombin to thrombin, or combine with thrombin to make it inactive. The normal thrombin generation test obtained with the patient's plasma contrasted markedly with the results of thrombin generation tests performed on normal plasma to which heparin had been added in sufficient amounts to render it incoagulable (Fig. 3). Moreover, this inhibition of thrombin formation was seen with both animal heparin and human " heparin."

The results of mixing the patient's plasma in varying amounts with normal plasma showed that the patient's plasma did not have the property of significantly prolonging the clotting time of normal plasma, except when the patient's plasma was present in large excess. This also is in marked contrast to blood to which heparin has been added in sufficient amounts to render it incoagulable, since such blood will cause considerable prolongation of the clotting time of normal blood.

Meneghini (1951) showed a correlation between the number of mast cells in the tissues and heparin activity of the blood in cases of leukaemia, liver disease, etc. In the present case there was no increase in the tissue mast cells either in the patient or the foetus.
In view of these findings it appears that, although the defect was reversible by protamine sulphate and toluidine blue, it was nevertheless not due to hyperheparinaemia. As a corollary it may be stated that reversibility by protamine sulphate and toluidine blue is not an adequate criterion for the diagnosis of hyperheparinaemia. Both protamine and toluidine blue are strongly tasic substances which may react with many acidiz macromolecules other than heparin.

Possible Site of Action of the " Anticoagulant." - Normally the conversion of fibrinogen to fibrin under the action of thrombin takes an appreciable time, and the action of thrombin is considered to be enzymatic in nature (e.g., Eagle, 1935 ; Morrison, 1947). Biggs and Macfarlane (1957) suggest that the formation of fibrin from fibrinogen involves three stages. First, acidic groups are spiit off from fibrinogen under the enzymatic action of thrombin. Secondly, the molecules thus formed polymerize, and thirdly under physiological conditions the fibrin so formed is strengthened by other linkages.

The fact that when protamine sulphate was added to the patient's uncitrated plasma in sufficient quantities fibrin appeared almost immediately suggests that the process of conversion of fibrinogen to fibrin under the action of thrombin had progressed at least part of the way, and that the "anticoagulant" was acting at some point in the reaction fibrinogen $\rightarrow$ fibrin, perhaps preventing the proper polymerization of the molecules.

The results shown in Fig. 2, where the addition of increasing amounts of protamine sulphate to the patient's plasma increased the size of the clot formed, suggest that there was a stoichiometric relationship between the protamine and its combination with the "anticoagulant."

Although the abnormality has been referred to as an "anticoagulant" the findings would be equally explicable on the basis of some alteration in the fibrinogen molecule itself, the abnormality being reversible by the addition of the strongly basic substances protamine sulphate or toluidine blue. The failure of the patient's plasma significantly to prolong the clotting time of normal plasma, and the fact that in the mixing experiments the amount of fibrin formed was proportional to the amount of normal plasma present (Fig. 2), are also in favour of this hypothesis of "altered" fibrinogen.

Frick (1955) described the occurrence of a plasma factor which inhibited the conversion of fibrinogen to fibrin in cases of multiple myelomatosis. In three cases the whole blood clotting 
time ranged from 13 to 25 minutes, and the thrombin time was prolonged. On electrophoresis an inhibitor was found which migrated with the $\beta$ and $\gamma$ globulin fractions. The inhibitor had an effect on normal plasma, and its action was apparently not counteracted by protamine. It therefore appears that the inhibitor in these cases was different from the one described here.

"Afibrinogenaemia" and "Altered" Fibrinogen. - The commonest clotting disorder associated with pregnancy is the so-called "hypo" or " afibrinogenaemia" (Moore, 1954), and the occurrence of a prolonged clotting time in our patient first suggested the diagnosis of "afibrinogenaemia."

Recently it has been shown that so-called " afibrinogenaemia" may not necessarily be associated with low levels of fibrinogen (Sharp, Howie, Biggs, and Methuen, 1958). These authors studied four cases of this syndrome and found that in three cases plasma fibrinogen levels, as estimated biochemically, were not such as would usually be considered dangerously low $(250,148$, and $298 \mathrm{mg}$. per $100 \mathrm{ml}$. respectively). As a result of their study, Sharp et al. suggest that in the early stages of this syndrome poor reactivity of fibrinogen to thrombin may be an important feature of the coagulation defect. These authors studied the effects of toluidine blue in vitro in their cases, and found it to be without effect. However, no mention is made of the concentrations used, or of any study of the effect of protamine sulphate. It is interesting that their cases also had thrombocytopenia.

Other Features.-It is noteworthy that the foetus was also affected in the same way as its mother, showing that the "anticoagulant" concerned could readily pass the placental barrier. This transplacental passage is perhaps further evidence that we are dealing with an actual anticoagulant rather than an abnormality of the fibrinogen molecule itself.

The presence of thrombocytopenia is an interesting though unexplained feature which may have contributed to the haemorrhagic state. It is interesting to note that Copley (1948) has suggested that the thrombocytopenia in subjects exposed to atomic bomb radiation may be due to increase in the blood "heparin." It may be that the thrombocytopenia in this patient was a secondary effect following on the presence of the " anticoagulant" and severe haemorrhage.

Treatment.-The tests in vitro suggested that protamine sulphate and toluidine blue would correct the abnormality. Unfortunately only a small amount of protamine was available and it took some hours to get toluidine blue. However, both these substances definitely improved the clotting mechanism. The patient died in the middle of the infusion of toluidine blue, but it is pure conjecture to speculate whether or not some reaction to the toluidine blue (which was only a laboratory stain and possibly not very pure) hastened her death.

The results of the mixing tests (Fig. 2) suggest that there was little or no excess " anticoagulant" free in the plasma, and it seems probable that the patient would have responded well to the administration of fibrinogen, or concentrated plasma had either of these been available. In the present state of our knowledge such a response might have been taken as a further pointer in .. favour of a diagnosis of " afibrinogenaemia."

We wish to thank Dr. D. Paranjothi, under whose care the patient was admitted, for asking us to see the patient and for permission to publish this report; Dr. E. Gault for the post-mortem findings and histological examinations; Miss M. Nash for the biochemical estimation of plasma fibrinogen; Dr. Ian Hansen for his help and advice ; Miss I. Ponnuswamy for preparing the human heparin; and Mr. S. P. Swaminathan for technical help.

\section{REFERENCES}

Allen, J. G., Sanderson, M., Milham, M., Kirschon, A., and Jacobson, L. O. (1948). J. exp. Med. 87, 71.

Bell, W. N. (1951). Blood, 6, 1199.

Biggs, R., and Macfarlane, R. G. (1957). Human Blood Coagulation and its Disorders, 2nd ed. Blackwell Scientific Publications, Oxford.

Douglas, A. S., and Macfarlane, R. G. (1953). J. Physiol. (Lond.), $122,554$.

Castex, M. R. (1946). Bull. Acad. Méd. (Paris), 130, 596.

and Pavlovsky, A. (1947). Sang, 18, 1.

Conley, C. L., Hartmann, R. C., and Morse, W. I. (1949). Bull. Johns Hopk. Hosp., 84, 255.

Copley, A. L. (1948). J. Amer. med. Ass., 137, 145.

Crisalli, M., and Cotellessa, G. (1951). In Proc. Third International Congress of the International Society of Hematology, Cambridge, 1950, p. 452 . Grune \& Stratton, New York.

Dacie, J. V. (1956). Practical Haematology, 2nd ed. Churchill, London.

Dieckmann, W. J. (1936). Amer. J. Obstet. Gynec, 31, 734.

Dreskin, O.H., and Rosenthal, N. (1950). Blood, 5, 46.

Eagle, H. (1935). J. gen. Physiol., 18, 547.

Frick, Paul G. (1955). Amer. J. clin. Path. 25, 1263.

General Medical Council (1948). The British Pharmacopoeia, 1948, p. 220. Constable, London.

Howell, W. H., and Holt, E. (1918). Amer. J. Physiol., 47, 328.

Jaques, L. B., and Waters, E. T. (1941). J. Physiol. (Lond.), 99, 454.

Klein, P. D., and Seegers, W. H. (1950). Blood, 5, 742.

Lyttleton, J. W. (1950). Thesis for Degree of Ph.D. in the University of London. Biophysical Studies on Thrombin and Antithrombin and the Kinetics of Their Reaction.

Madison, F. W., and Quick, A. J. (1945). Amer. J. med. Sci., 209, 443.

Mellanby, J. (1934). Proc. roy. Soc. B., 116, 1.

Meneghini, P. (195i). Proc. Third Int. Congress of the International Society of Hematology, Cambridge, 1950, p. 505. Grune \& Stratton, New York.

Moore, H. C. (1954). Brit. med. J., 2, 277.

Morrison, P. R. (1947). J. Amer. chem. Soc., 69, 2723.

Quick, A. J. (1938). Amer. J. Physiol., 123, 712.

Sharp, A. A., Howie, B., Biggs, R., and Methuen, D. T. (1958) Lancet, 2, 1309. 